\title{
Towards a methodology for the characterisation of the fabric of wet clays using X-ray scattering
}

\author{
Georgios Birmpilis ${ }^{1, *}$, Reza Ahmadi-Naghadeh ${ }^{1}$, and Jelke Dijkstra ${ }^{1}$ \\ ${ }^{1}$ Chalmers University of Technology, Dep. of Arch. and Civil Eng.,SE-41296, Gothenburg, Sweden
}

\begin{abstract}
X-ray scattering is a promising non-invasive technique to study evolving nano- and micromechanics in clays. This study discusses the experimental considerations and a successful method to enable $\mathrm{X}$-ray scattering to study clay samples at two extreme stages of consolidation. It is shown that the proposed sample environment comprising flat capillaries with a hydrophobic coating can be used for a wide range of voids ratios ranging from a clay suspension to consolidated clay samples, that are cut from larger specimens of reconstituted or natural clay. The initial X-ray scattering results using a laboratory instrument indicate that valuable information on, in principal evolving, clay fabric can be measured. Features such as characteristic distance between structural units and particle orientations are obtained for a slurry and a consolidated sample of kaolinite. Combined with other promising measurement techniques from Materials Science the proposed method will help advance the contemporary understanding on the behaviour of dense colloidal systems of clay, as it does not require detrimental sample preparation
\end{abstract}

\section{Introduction}

Fine-grained soils, such as clay, remain a challenging material in many geotechnical settings. Consequently, the study of hydro-mechanical behaviour of natural clay and their artificial counter parts is still ongoing. In the last two decades or so, new experimental methodologies have been introduced to study the micro-structural origin of the emerging behaviour at continuum scale for predominantly coarse grained soils. Especially, imaging methods that allow to fully resolve the three-dimensional structure and shape of the assembly prove to be invaluable, e.g. [1]. The latter methods are not easy to replicate for fine-grained soils (grain size $<2 \mu \mathrm{m}$ ). Furthermore, the interactions on the particle scale are not solely frictional. Rather, the small particle size combined with their nanometre thickness results into an electrically charged particle. Such particles form complex colloid structures when dispersed in water. Their diminutive size challenge even the most advanced measurement methods, therefore, there is only limited data reported in literature for these materials. Especially data is scarce on the fabric of clays in their natural wet state. New observations at the governing microscopic scale (nm to $\mu \mathrm{m}$ ), hence, can offer valuable information on the emerging behaviour of the material at continuum scale. This paper discusses an experimental method for the preparation of samples for subsequent X-ray scattering experiments for the study of the fabric in wet clay samples.

$\mathrm{X}$-ray diffraction was used first as a mineral identification technique, i.e., the main focus was to study the micro-structural composition of the clay[2, 3]. Moreover, traditional experimental methods such as direct space mi-

*e-mail: georgios.birmpilis@ chalmers.se croscopy (e.g., Scanning Electron Microscopy) and Mercury Intrusion Porosimetry (MIP) [4-8, 15] have been extensively used to investigate particle shape and size of clay minerals, as well as the inter-particle fabric. These methods have been offering valuable insight in micro-structure of clay. They are, however, constrained by the required processes of sample preparation such as the pore fluid replacement and the surface treatment. Recent research indicates that some of those preparation techniques are intrusive, as the natural configuration of the fabric is altered [9]. Non-invasive techniques are able to mitigate these limitations, as well as offering the opportunity to study the evolving fabric by combining continuous mechanical probing with simultaneous monitoring. Scattering is a family of experimental techniques suitable for nonintrusive methods to study colloidal systems such as finegrained soils. Scattering is an extension of diffraction and yields additional information on the chemical composition, the particle morphology and orientation. A major advantage is that there is no need for detrimental preparation procedures that can alter the micro-structure of the specimen. Scattering has been previously used for the study of low concentration single mineral clay suspensions [1012]. Dense solid colloid systems, such as consolidated clay, however, have not been systematically probed using scattering methods.

\section{Experimental Methodology}

The feasibility of scattering techniques has been evaluated for the study of fabric change in clay samples over a wide range of void ratios that represent geomechanical relevant conditions. Therefore, the experiments have been 
performed on a clay sample in slurry state and a sample prepared from consolidated slurry. The clay mineral used is kaolinite.

\subsection{Experimental considerations}

Some general experimental considerations related to scattering experiments on the clay samples will be discussed first, before further elaboration for the current test series on a slurry and consolidated sample of kaolin using X-ray scattering.

- Selection of sample environment. Depending on the instrument used the sample holder has to operate in vacuum, a separate sample environment facility, or free standing. Furthermore, plane strain samples need to be prepared to accommodate the measurement technique. Finally, the front and back wall should be X-ray (or neutron) transparent, so that a rather thick clay sample can be accommodated. In order to prevent edge effects and wall effects (in the sample, as the signature of the empty sample holder will be compensated for in the measurement) the sample size is $1 \mathrm{~mm}$, i.e. substantially larger than for microscopy, but smaller than any other geotechnical test specimen. Also, the samples need to be sealed to prevent drying and other unwanted effects.

- Sample preparation. Samples can be either reconstituted in the sample holder, or alternatively directly cut in the sample holder out of a larger sample. The latter proves to be most repeatable and compatible with intact natural samples. Also, reconstitution of miniature samples, e.g. in a small diameter capillary, proves challenging due to the wall friction effects.

- X-ray scattering measurement. In order to get best results, the composition of the material, hence $d$-spacing of its constituents, should be known a priori (e.g. from diffraction). This allows to configure the sample - detector distance, although modern instruments are able to change this automatically. The largest length scale that can be accessed is generally $<200 \mathrm{~nm}$ (USAXS). The experimenter can choose for single point measurements or a raster scan, where for each in plane coordinate a depth averaged scattering signal is collected. Depending on the brightness of the source, the sample dimensions (hence attenuation) and acquisition settings required to obtain signals above the noise floor this takes less than a minute (synchrotron source) to several hours (lab instrument).

- Interpretation of results. The interpretation of scattering data of colloid systems with a large concentration (consolidated clay) is non-trivial, as most models for back-analysis are based on idealised geometries of particles in suspension. Partial shading effects from overlapping grains and varying pore throat sizes are not captured. Typically, only strongly organised re-occurring structural features are detected.

\subsection{Sample preparation}

The experiments are performed on a slurry and consolidated specimen reconstituted from kaolinite powder. The material has well known physico-chemical properties and the composition, hence associated $d$-spacing, is known. Furthermore, as opposed to natural clays, the specimen will not have impurities that attenuate the signal unexpectedly. The samples are reconstituted from a slurry at $100 \%$ water content $\left(1.5 w_{L}[13]\right)$. The kaolinite powder $\left(G_{s}=2.6\right)$ is first mixed with dimineralised water with $\mathrm{pH}=7$ and subsequently de-aired under vacuum. For the consolidated sample the slurry was consolidated in an oedometer cell, up to a vertical stress level $\sigma_{v}^{\prime}$ of $65 \mathrm{kPa}$ before being unloaded for subsequent sub-sampling.

In the current study flat capillaries made out of borosilicate glass (Hilgenberg $\mathrm{GmbH}$ ) are used in order to maintain the initial water content. The slurry was directly poured into the capillary and subsequently sealed. In the case of the consolidated sample the capillaries were used to cut the material directly in the capillary from the oedometer sample. The thin-wall capillaries (wall thickness of $0.16 \mathrm{~mm}$ and a cross section of $1.4 \mathrm{~mm}$ by $6.5 \mathrm{~mm}$ ) are pushed in the clay with a constant rate of penetration of $0.01 \mathrm{~mm} / \mathrm{min}$ to minimise the generation of excess pore pressures. An indication of good sampling is that the penetration depth equals the height of the specimen in the capillary, i.e. no plugging occurred. In order to reduce the adhesive component of the inner wall friction of the capillary, a dry hydrophobic coating is applied. After sampling the ends are sealed with first a grease lubricant and secondly silicon glue so prevent loss of water and make them air tight for vacuum conditions.

\subsection{X-ray scattering}

$\mathrm{X}$-ray Scattering is a non-destructive technique, which utilises the elastic scattering of X-rays to provide bulk measurements of the structure of scatterers in the $\AA$ to $\mathrm{nm}$ scale. The theoretical framework for analysis in SAXS is based on Bragg's Law [14]:

$$
n \lambda=2 d \sin \theta
$$

where, $d$ is the distance between atomic layers of a crystal structure, $\lambda$ is the wavelength of the incident $\mathrm{X}$ ray beam and $n$ is an integer. The interference of an incident X-ray with a crystal structure of characteristic $d$ spacing is constructive (producing a wave with the same phase, wavelength and double amplitude) when Bragg's law is satisfied. The term diffraction is commonly referred to in crystallography, for lattices with strict periodic arrangement of unit cells. Scattering uses the same principal but looks into larger length scales and thus smaller scattering angles. Very low scattering angles, $0.1^{\circ}-10^{\circ}$, allow capturing any heterogeneity in the nm-range (given this d-spacing is re-occurring throughout the measurement volume). Wide Angle X-ray Scattering (WAXS) falls inbetween Small Angle X-ray Scattering and diffraction. For even larger spacings, Ultra Small Angle X-ray Scattering (USAXS) should be employed. In scattering analysis instead of the scattering angle $\theta$, the scattering vector or momentum transfer $q$ is preferred.

The Chalmers Material Analysis Laboratory X-ray scattering instrument, Mat:Nordic SAXS/WAXS/GISAXS, 
has been used for the X-ray scattering measurements. The device enables Ultra Small, Small and Wide angle X-ray scattering configurations by changing the sample-detector distance. As a result the attainable $q$-range of the instrument ranges from $0.0025 \AA^{-1}$ up to $2.8 \AA^{-1}$, or $d$-spacings of 2.24 to $2512 \AA$ respectively. The detector is a $\mathrm{Pi}$ latus $300 \mathrm{~K}$. The hutch is kept under vacuum conditions during the measurements, to eliminate X-ray attenuation from scattering of air particles. For quantitative comparisons between different samples, all measurement results are normalised with the initial intensity readings without sample in place. Furthermore, any effects of the sample holder are compensated by subtracting the measurement of the empty capillary from the signal with filled capillary.

\section{Results}

Figure 1:left presents the Ultra Small Angle X-ray Scattering curve for the kaolinite slurry. As discussed in the previous Section, all measurements are normalised and compensated for the sample holder effects. The results show no peak that indicates a characteristic spacing is observed on this observation scale. The maximum $d$-spacing that can be observed in the USAXS configuration of this instrument is $200 \mathrm{~nm}$. This result is to be expected, since the large dimension of the kaolinite particles is more than $5 \mu \mathrm{m}$ and the particles in a slurry with this high void ratio are not in a dense configuration. As a result, the measurement range in the USAXS configuration is unable to detect the larger length scales associated to the plateletplatelet structure whilst being not sufficiently sensitive to detect the smaller $d$-spacings within the clay mineral. At wider angles, hence higher resolving power of small $d$ spacings, the crystal structure of the minerals appear in the WAXS curves (Figure 1:right). The main mineral identified at $q=0.886 \AA^{-1}$ is kaolinite which has a documented $d$-spacing of $7 \AA$. The secondary peak at $q=0.637 \AA^{-1}$ or $d=10 \AA$ corresponds to illite. These measurements corroborate the description of the manufacturer of the kaolinite powder on $95 \%$ purity (the peaks appear magnified on the semi-logarithmic scale). The increase of intensity for the case of consolidated kaolin is due to the higher number of scatterers contained in the illuminated volume from the $\mathrm{X}$-rays. Hence, the lower voids ratio (higher density) is linked to higher scattering intensities.

Additionally to obtain information on structural spacing, SAXS provides insight into the distribution of orientation of all the scatterers -clay particles in this case- through the path of X-rays. This is achieved by plotting the intensity of photons at a constant $q$ (hence $d$-spacing) as function of the azimuth on the 2D detector. Higher intensity in specific azimuth range signifies a preferred orientation of particles for this azimuth. Here, the azimuth $q$ corresponding to the kaolinite mineral is plotted, where the horizontal orientation refers to an azimuth of 0 degrees (Figure 2). In addition to the scattering measurements, also the corresponding orientations of the particles are sketched on top of the Figure. The kaolinite particles are randomly distributed in the slurry mixture for which an equal intensity as function of azimuth is measured. In contrast, the mineral is strongly aligned towards the horizontal direction after the one-dimensional consolidation stage, this is in line with the findings reported in [15]. The discontinuities in the signal relate to the design of the detector panel which has some gaps where multiple sensing elements are combined.

The fact that the clay is in its unaltered state allows, in principle, to monitor the consolidation process in-situ (within the instrument) under mechanical loading with simultaneous scattering observations. Furthermore, some preliminary results have already been performed on intact samples of natural clay with promising results. The complexity of the sample, however, makes direct interpretation of the processes more difficult, as for the sensitive clay tested the minerals are not stable.

\section{Conclusions}

This paper discusses the experimental considerations and a successful method to enable X-ray scattering to study clay samples at two extreme water content levels. It is shown that the proposed sample environment, comprising flat capillaries with a hydrophobic coating, can be used for a wide range of void ratios ranging from a clay suspension to consolidated clay samples, that are cut from larger specimens of reconstituted (hence natural) clay. The initial X-ray scattering results using a laboratory instrument indicate that valuable information on, in principal evolving, clay fabric can be obtained. For the samples tested the Wide Angle X-ray Scattering measurement range that covers the $d$-spacing of the clay minerals was most informative. The USAXS range ( $d$-spacing up to $200 \mathrm{~nm}$ ) was insufficient to detect any repeating length scales at the agglomeration scale. Features such as characteristic distance between structural units and particle orientations are obtained for a slurry and a consolidated sample of kaolinite: the disperse structure in the slurry changed in a highly horizontally aligned structure after consolidation.

\section{References}

[1] S. A Hall , M. Bornert, J. Desrues, Y. Pannier, N. Lenoir, G. Viggiani, P. Bésuelle (2010). Discrete and continuum analysis of localised deformation in sand using X-ray [mu] CT and volumetric digital image correlation. Géotechnique 60,315-322.

[2] R.E. Grim, Clay Mineralogy, (McGraw Hill, New York, 1953)

[3] G.W. Brindley, Sari .S. Kurtossy (1962). Quantitative determination of Kaolinite by $X$-ray diffraction.The American Mineralogist 47,1213-1215.

[4] S. Diamond (1970). Micro-structure and pore structure of impact compacted clays. Clay and Clay Minerals 19, No.4, 239-249.

[5] J.E. Gillot (1970). Fabric of Leda clay investigated by optical, electron-optical, and X-ray diffraction methods. Engineering Geology 4, No. 2, 133-153. 

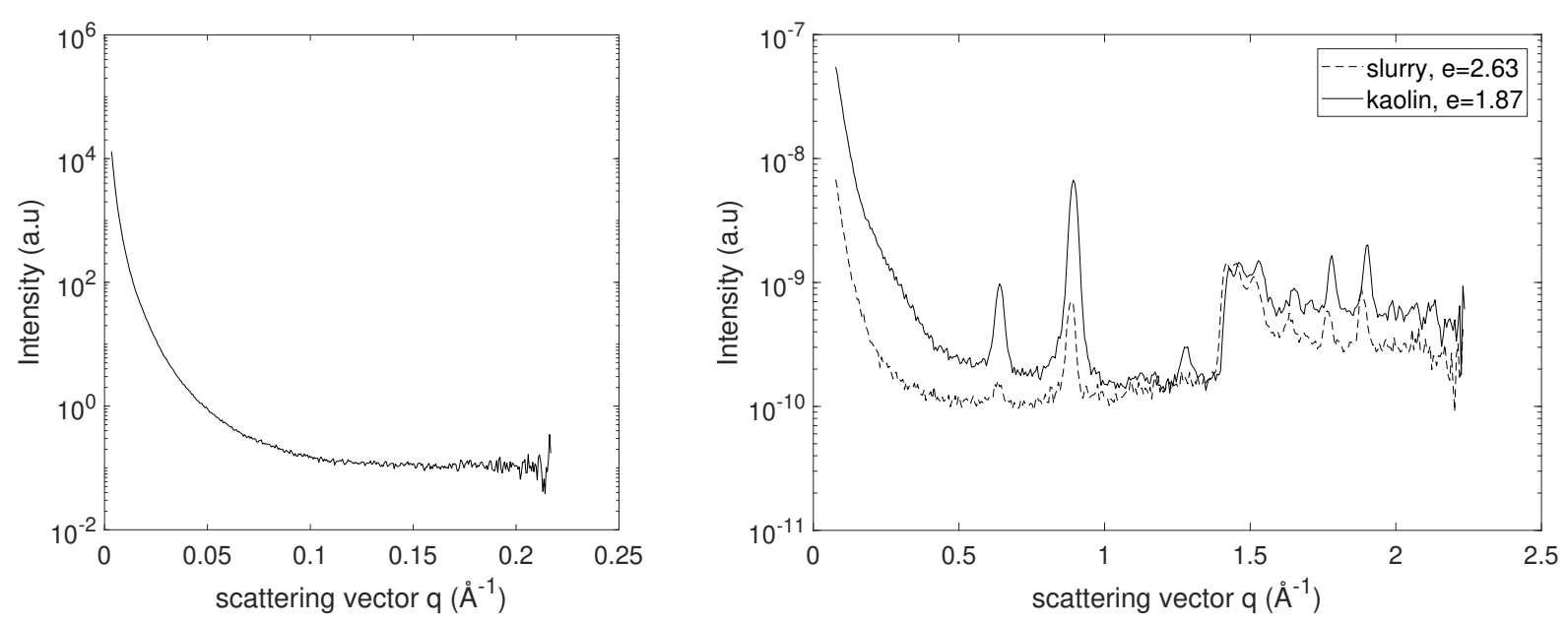

Fig. 1. Left: Ultra Small Angle X-ray Scattering curve for kaolinite slurry at $100 \%$ water content $(e=2.6)$; Right: Wide Angle X-ray Scattering curve for the slurry and the consolidated sample.

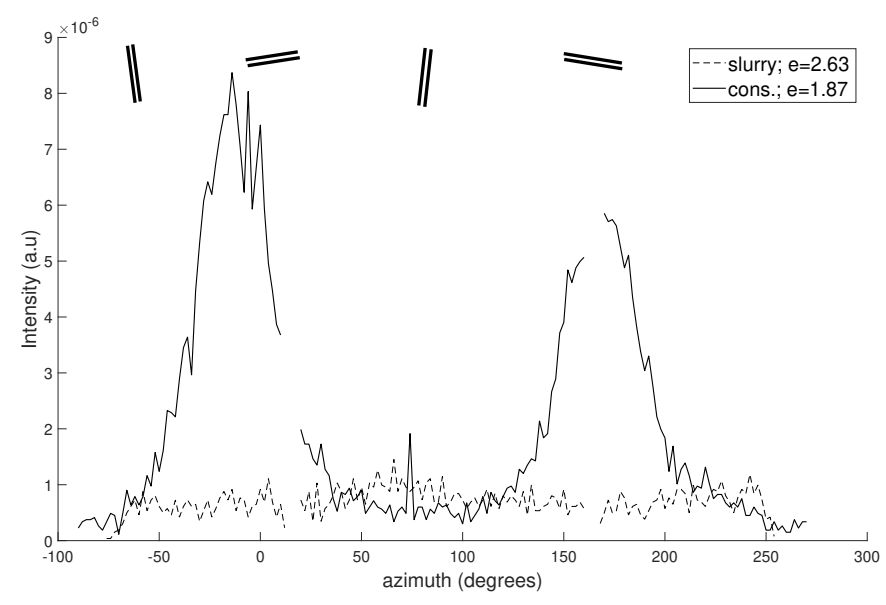

Fig. 2. Distribution of the orientation of the scatterers in two states of kaolin: slurry and consolidated. Note the sketched particle orientations at the top of the graph.

[6] R. Pusch (1970). Microstructural change in soft quick clay at failure. Can. Geotech. J. 7, No. 1, 1-7.

[7] P. Delage, G. Lefebvre (1984). Microstructural analysis of inherent and induced anisotropy in clay. Canadian Geotechnical Journal 91,21-35.

[8] Y-H. Wang, W-K. Siu (2006). Structure characteristics and mechanical properties of kaolinite soils. I. Surface charges and structural characterizations. Canadian Geotechnical Journal 43,587-600.

[9] A. Deirieh, I.Y. Chang, M.L. Whittaker, S. Weigand, D. Keane, J. Rix, J.T. Germaine, D. Joester, P.B. Flemings (2018). Particle arrangements in clay slurries: The case against the honeycomb structure.Applied Clay Science 152,166-172.

[10] H.J.M. Hanley, G.C. Straty, F. Tsvetkov (1994). A Small Angle Neutron Scattering Study of a Clay Suspension under Shear. Langmuir 10 No.9, 3362-3364.

[11] M. Morvan, D. Espinat, J. Lambard, Th. Zemb (1994). Ultrasmall- and small-angle X-ray scattering of smectite clay suspensions. Colloids and Surfaces A: Physicochemical and Engineering Aspects 82 No.2, 193-203.

[12] L. Zhang, C. Jahns, B.S. Hsiao, B. Chu (2003). Synchrotron SAXS/WAXS and rheological studies of clay suspensions in silicone fluid. Journal of Colloid and Interface Science, 266 No.2, 339-345.

[13] N.K. Toker, R. Ahmadi-Naghadeh (2018). A New Isotropic Specimen Preparation Method from Slurry for both Saturated and Unsaturated Triaxial Testing of a Low-Plasticity Silt. Journal of Colloid and Interface Science, 42 No.4.

[14] A. Guinier, G. Fournet, Small Angle Scattering of $X$ rays (3rd edn. John Wiley \& Sons, New York, 1955)

[15] P.Y. Hicher, H Wahyudi, D. Tessier (2000). Study of the structure of a sensitive Champlain clay and of its evolution during consolidation. Mechanics of Cohesive-frictional Materials 5,341-371. 